\title{
MicroRNA expression profiles of LO2 cells expressing the wild-type and mutant HBx gene
}

\author{
XIAOYU FU ${ }^{1}$, DEMING TAN ${ }^{1}$, ZHOUHUA HOU ${ }^{1}$, ZHILIANG HU ${ }^{2}$, GUOZHEN LIU $^{1}$, YI OUYANG ${ }^{1}$ and FEI LIU \\ ${ }^{1}$ Department of Infectious Diseases, Xiangya Hospital, Central South University, Changsha, Hunan 410008; \\ ${ }^{2}$ Department of Infectious Diseases, The Second Hospital of Nanjing, Nanjing, Jiangsu 210000, P.R. China
}

Received July 21, 2012; Accepted November 7, 2012

DOI: $10.3892 / \mathrm{mmr} .2012 .1203$

\begin{abstract}
Hepatocellular carcinoma (HCC) is one of the most common malignancies worldwide. Although numerous studies have suggested the potentially oncogenic roles of wild-type or mutant hepatitis $\mathrm{B}$ virus $\mathrm{X}(\mathrm{HBx})$ protein in hepatocarcinogenesis, their exact mechanism remains unclear. Increasing evidence suggests that microRNAs (miRNAs) play essential roles in embryogenesis, cell differentiation and carcinogenesis. This study aimed to investigate the effect of $\mathrm{HBx}$ on the miRNA expression profile of LO2 cells. We established the LO2 cell line transfected with recombinant plasmid pcDNA3.0/HBx-d382, pcDNA/HBx and plasmid pcDNA3.0 using Lipofectamine ${ }^{\mathrm{TM}}$ 2000, which was confirmed by reverse transcription-polymerase chain reaction (RT-PCR) and western blotting. We then demonstrated the miRNA expression profiles in the stably transfected LO2 cells using a mammalian miRNA microarray containing whole-human mature and precursor miRNA sequences. The results were confirmed by real-time quantitative PCR (qPCR). RT-PCR and western blot analysis showed that a stably HBx-transfected LO2 cell line had been successfully established. According to the microarray, compared to LO2/pcDNA3.0 cells, 6 miRNAs were shown to have higher expression and 5 were shown to have decreased expression in $\mathrm{LO} 2 / \mathrm{HBx}-\mathrm{d} 382$ cells, while 4 up- and 12 downregulated miRNAs were observed in LO2/HBx cells. There were 8 different expression patterns of miRNAs between LO2/HBx and LO2/HBx-d382 cells. All the chip results were consistent with the real-time PCR data. Consequently, the HBx gene may influence the miRNA expression profile of $\mathrm{LO} 2$ cells. Thus, it may be helpful to further investigate the role of $\mathrm{HBx}$ in hepatocarcinogenesis and clarify the underlying molecular mechanisms involved.
\end{abstract}

Correspondence to: Professor Deming Tan, Department of Infectious Diseases, Xiangya Hospital, Central South University, Xiangya Road, Kai Fu District, Changsha 410008, Hunan, P.R. China E-mail: demingtan@yeah.net

Key words: microRNA, hepatitis B virus X protein, hepatocellular carcinoma

\section{Introduction}

Hepatocellular carcinoma (HCC) is one of the most common malignancies worldwide, accounting for 500,000-600,000 mortalities per year (1). It has been estimated that approximately $53 \%$ of $\mathrm{HCC}$ cases are related to hepatitis B virus (HBV) (2). HBV, which belongs to the Hepadnaviridae family, causes both acute and chronic infection of the liver. HBV is a partial double-stranded DNA virus with a 3.2-kb genome containing four known open reading frames, namely, S, C, $\mathrm{P}$ and $\mathrm{X}$ genes. The $\mathrm{X}$ gene, which encodes hepatitis $\mathrm{B}$ virus $\mathrm{X}$ protein $(\mathrm{HBx})$, is most closely associated with $\mathrm{HCC}$ (3). Evidence suggests that $\mathrm{HBx}$ is a multifunctional regulator that modulates the cell cycle, genetic stability, transcription, protein degradation, signal transduction and apoptosis by directly or indirectly interacting with host factors (4). The protein inhibits cell proliferation by inducing late G1 arrest and induces apoptosis in a p53-dependent and -independent manner (5). Several transgenic mouse models reveal that the $\mathrm{X}$ gene is capable of inducing HCC (6). Notably, a number of studies have demonstrated that mutations and deletions of $\mathrm{HBx}$, particularly the $\mathrm{COOH}$-terminal deletion of $\mathrm{HBx}$, are frequent events in $\mathrm{HBV}$-associated HCC tissues (7). It has been reported that mutations of the $\mathrm{HBx}$ gene may cause uncontrolled growth and contribute to multistep hepatocarcinogenesis (8). Therefore, mutations of the HBx gene are very important in hepatocarcinogenesis. However, the exact molecular mechanisms involved in hepatocarcinogenesis remain unclear.

MicroRNAs (miRNAs) are a class of short ( $\sim 22$ nucleotides), endogenous, single-stranded noncoding RNAs. They are responsible for the post-transcriptional expression regulation of targeted genes. Growing evidence shows that miRNAs may play a key role in the regulation of cellular differentiation, proliferation, apoptosis, gene expression and cancer development (9). HCC is a complex disease involving epigenetic and chromosomal instability, as well as expression abnormalities of both coding and noncoding genes; the latter include miRNAs (10). It has been found that miR-18 and miR-224 are significantly overexpressed and miR-195, miR-199a, miR-200a and miR-125a are underexpressed in HCC tissues (10). Furthermore, several miRNAs downregulated in HCC, such as miR-21, miR-221, miR-223 and miR-224, have been identified as modulators of cell growth, apoptosis, migration or inva- 
sion (11). miR-152 has also been found to be downregulated in HBV-related HCC tissues (12).

In a previous study, our data showed that a mutant of the HBx gene with a deletion from 382 to $400 \mathrm{bp}$ (HBx-d382) was a common event and potentially related to the development of HCC (13). However, the molecular mechanisms of HBx involved in HCC development are still not well understood. Moreover, given the importance of miRNAs in HCC development, we investigated whether miRNAs play a role in the hepatocarcinogenic effect of HBx. The present study investigated the miRNA expression profiles of the non-tumor human hepatic LO2 cells stably transfected with HBx and HBx-d382 using miRNA microarray.

\section{Materials and methods}

Cells culture and establishment of the stably transfected cell lines. The plasmid pcDNA3.0, recombinant plasmid pcDNA3.0/HBx-d382 (a mutant of the HBx gene with a deletion from 382 to $400 \mathrm{bp}$ ) and pcDNA/HBx (the restructuring of HBx genetic fragments originates from liver cell line HepG2.215) were all from our laboratory and were stably established previously (13).

The hepatocyte cell line LO2 (Chinese Academy of Science, Cell Biology of Shanghai Institute, Shanghai, China) was cultured in RPMI-1640 medium (Gibco, Carlsbad, CA, USA) containing $100 \mathrm{U} / \mathrm{ml}$ penicillin, $100 \mu \mathrm{g} / \mathrm{ml}$ streptomycin and $10 \%$ fetal bovine serum (FBS; Gibco) in a humidified atmosphere of $5 \% \mathrm{CO}_{2}$ and $95 \%$ air at $37^{\circ} \mathrm{C}$. We attempted to establish a stable LO2 cell line transfected with $\mathrm{HBx}$ and HBx-d382.LO2 cells were transfected with Lipofectamine 2000 (Invitrogen, Carlsbad, CA, USA) according to the manufacturer's instructions and selected with G418 (Geneticin ${ }^{\circledR}$; Gibco). The plasmids pcDNA3.0, pcDNA3.0/HBx-d382 and pcDNA/HBx were used in the transfection experiments. Empty pcDNA3.0 vector plasmid was used as a control. The stable transfection of pcDNA3.0/HBx-d382 (termed LO2/HBx-d382), pcDNA/HBx (termed $\mathrm{LO} 2 / \mathrm{HBx}$ ) or the empty vector (termed LO2/pcDNA) was confirmed by reverse transcription-polymerase chain reaction (RT-PCR) and western blot analysis. The study was approved by the ethics committee of Xiangya Hospital, Central South University, Changsha, China.

RT-PCR analysis. Total RNA of the LO2 cell line was extracted using TRIzol (Invitrogen). The primers used in the PCR were as follows: HBx, F, 5'-AAGGTACCATGGCT GCTAGGCTGTGCT-3' and R, 5'-CTGGGCCCTTAGGCA GAGGTGAAAAAGTTG-3' (481 or 462 bp amplified fragment); $\beta$-actin, F, 5'-CTCCATCCTGGCCTCGCTGT-3' and R, 5'-GCTGTCACCTTCACCGTTCC-3' (242 bp amplified fragment). The cycling conditions for $\mathrm{HBx}$ amplification were $94^{\circ} \mathrm{C}$ for $3 \mathrm{~min}$, followed by 35 cycles of $94^{\circ} \mathrm{C}$ for $30 \mathrm{sec}, 68^{\circ} \mathrm{C}$ for $30 \mathrm{sec}$ and $72^{\circ} \mathrm{C}$ for $1 \mathrm{~min}$, followed by an elongation cycle of $72^{\circ} \mathrm{C}$ for $5 \mathrm{~min}$. The resultant PCR products were analyzed using electrophoresis on $1.5 \%$ Tris/Borate/EDTA (TBE) agarose gel with $\beta$-actin as an internal control.

Western blot analysis. Total protein was extracted from transfected cells using the RIPA lysis buffer (Beyotime, Nantong, China). Equal amounts of protein samples were separated
Table I. Corresponding absorbance at 260 and $280 \mathrm{~nm}$.

\begin{tabular}{lccc}
\hline Cells & A260/A280 & 2100 RIN & 28s $/ 18 \mathrm{~s}$ \\
\hline L02/pcDNA3.0 & 1.99 & 10 & 2.2 \\
L02/HBx & 2.11 & 10 & 2 \\
L02/HBx & 1.97 & 10 & 2.2 \\
\hline
\end{tabular}

by SDS-PAGE and electroblotted onto PVDF membranes (Millipore, Billerica, MA, USA). Blots were blocked with 5\% skimmed milk, followed by incubation with antibodies specific for rabbit anti-HBx (dilution, 1:1,500; Abcam, Cambridge,UK), and mouse anti- $\beta$-actin (dilution, 1:10,000; Sigma, St. Louis, MO, USA). Blots were then incubated with goat anti-rabbit or anti-mouse secondary antibody conjugated to horseradish peroxidase (dilution, 1:5,000; Jackson ImmunoResearch, Inc., West Grove, PA, USA) and visualized by enhanced chemiluminescence (ECL) (Amersham Biosciences, Piscataway, NJ, USA).

miRNA isolation. Total RNA of LO2 cells transfected with wild-type and mutant $\mathrm{HBx}$ as well as empty plasmid pcDNA3.0 was extracted using TRIzol. The concentration and quality of RNA was measured by ultraviolet (UV) absorbance at 260 and $280 \mathrm{~nm}$ and checked by gel electrophoresis individually. miRNA isolation was performed from all pooled total RNA using an miRNA isolation kit (Ambion, Austin, TX, USA). RNA quality was measured using the Small RNA kit (Agilent, Santa Clara, CA, USA) on the Agilent 2100 Bioanalyzer and analyzed by capillary electrophoresis individually. The average RNA integrity number (RIN) value of all samples was $\geq 6.0$ and $28 \mathrm{~s} / 18 \mathrm{~s}>0.7$, indicating high-quality RNA with minimal degradation products.

miRNA microarray analysis. The Human miRNA Microarray version 3.0 (Agilent) contains approximately 15,000 probes including 866 human and 89 human viral miRNAs from the Sanger database v12.0. This chip not only has high sensitivity and specificity but can also distinguish between mature and precursor miRNAs. Microarray analysis was performed according to the manufacturer's instructions (Agilent). Four clones of each cell line were analyzed with microarray analysis. Briefly, first strand cDNA was synthesized from $200 \mathrm{ng}$ RNA, followed by cRNA amplification and labeling with $\mathrm{Cy} 3$ or Cy5. Equal amounts of Cy3- or Cy5-labeled cRNA from the cells of the LO2/HBx-d382, LO2/HBx or LO2/pcDNA3.0 fraction were mixed and hybridized using the Agilent Human Whole Genome Oligo Microarray in a dye swap design at $65^{\circ} \mathrm{C}$ overnight. On the following day, slides were washed and signals were scanned with GenePix ${ }^{\mathrm{TM}}$ 4000B (Agilent). There were four spot replicates for each probe on the chip.

Signal intensities from scanned images were analyzed using Agilent Feature Extraction software version 10.5 and GeneSpring GX. The abundance of probes in the cells of the LO2/HBx-d382, LO2/HBx or LO2/pcDNA3.0 fraction was compared with the abundance in the total fraction. For each probe, the signal intensity from the cells of the $\mathrm{LO} 2 / \mathrm{HBx}-\mathrm{d} 382$, LO2/HBx or LO2/pcDNA3.0 fraction was divided by the 

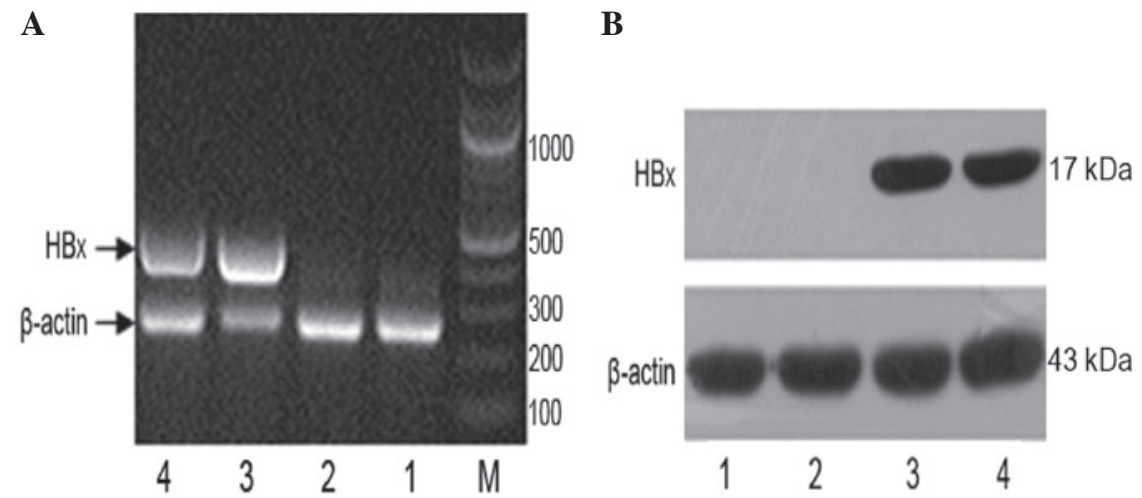

Figure 1. Identification of stable HBx transfection in LO2 cells. (A) Integration of the HBx gene was identified by RT-PCR. (B) Western blot analysis showed the expression of HBx in LO2 cells. 1, LO2; 2, LO2/pcDNA3.0; 3, LO2/HBx-d382; 4, LO2/HBx; M, marker. HBx, hepatitis B virus X protein.

signal intensity from the total cell lysate RNA fraction. For each dye swap, the average LO2/HBx-d382/T, LO2/HBx/T or $\mathrm{LO} 2 / \mathrm{pcDNA} 3.0 / \mathrm{T}$ ratio was calculated. Fluorescence intensity was normalized with the background subtracted, and all scanned images were processed and converted into normalized data using a quantile method. LO2/HBx-d382/T and $\mathrm{LO} 2 / \mathrm{HBx} / \mathrm{T}$ of each probe were compared with each other as well as with LO2/pcDNA3.0/T. After standardization, we output the $\log 2$ value of the primary signal. Two criteria were set to identify the abnormal expression of miRNAs: when compared with LO2/pcDNA3.0, a different value of each probe present in the LO2/HBx-d382 or LO2/HBx $>1$ (corresponding to the ratio of the original signal is $>2$ ) was considered to be enriched in the LO2/HBx-d382 or LO2/HBx fraction; while a different value $<-1$ (corresponding to the ratio of the original signal is $<0.5$ ) was considered to be reduced in $\mathrm{LO} 2 / \mathrm{HBx}-\mathrm{d} 382$ or $\mathrm{LO} 2 / \mathrm{HBx}$ fraction.

Real-time PCR. Total RNA of transfected cells was extracted using TRIzol (Invitrogen) according to the manufacturer's instructions. cDNA was synthesized with the RevertAid ${ }^{\mathrm{TM}}$ First Strand cDNA Synthesis kit (MBI Fermentas, Inc., Burlington, ON, Canada) in a total volume of $20 \mu l$. The cDNA sample was analyzed by real-time PCR using the THUNDERBIRD ${ }^{\circledR}$ SYBR qPCR mix (Toyobo, Osaka, Japan). All the primers of miRNAs that were detected as differentially expressed in the microarray were from a Bulge-Loop ${ }^{\mathrm{TM}}$ miRNA qPCR Primer Set (RiboBio, Guangzhou, China) including the human U6 snRNA. The experiment consisted of two steps: i) it was based on the stem-loop structure protruding primer reverse transcription reaction and ii) real-time fluorescence quantitative PCR (qPCR). The stem-loop structure of the RT-convex primers combined with miRNA 3 ' terminal end and the reverse transcriptase leads to the reverse transcription reaction, while the specific primers and SYBR Green fluorescent dye participating in the quantitative PCR reaction system realizes quantitative detection of reverse transcription products. Each cDNA sample was run on a 96-well optical plate in a total volume of $20 \mu \mathrm{l} /$ well including $2 \mu \mathrm{l}$ RT product, $4 \mu \mathrm{l}$ specific primers, $4 \mu \mathrm{l}$ sterile double-distilled water (ddw) and $10 \mu \mathrm{l}$ SYBR qPCR Mix. qPCR was performed on the 7500HT Real-Time PCR System (Applied Biosystems, Foster City, CA, USA) using the following profile: $95^{\circ} \mathrm{C}$ for $1 \mathrm{~min}$, and 40 cycles of $95^{\circ} \mathrm{C}$ for $15 \mathrm{sec}$ and $60^{\circ} \mathrm{C}$ for $35 \mathrm{~min}$. A dissociation step was performed following the qPCR amplification for melting curve analysis. All reactions were performed in triplicate. The whole miRNA expression of LO2/HBx-d382 or LO2/HBx cells were compared to each other as well as to LO2/pcDNA3.0 cells and normalized using U6 snRNA. Since a cycle threshold (CT) value of 35 represents single molecule template detection, CT values $>35$ were considered to be below the detection level of the assay. Therefore, only the miRNAs with CT $\leq 35$ were included in the analyses.

The relative amount of miRNAs studied in the samples was determined with the $2^{-\Delta \Delta \mathrm{CT}}$ method, where $\left.\Delta \Delta \mathrm{CT}=\mathrm{CT}_{\text {target }}-\mathrm{CT}_{\mathrm{U} 6}\right)_{\text {sample }}-\left(\mathrm{CT}_{\text {target }}-\mathrm{CT}_{\mathrm{U} 6}\right)_{\text {control }}$. Briefly, the $\mathrm{CT}$ values for the U6 snRNA were subtracted from $\mathrm{CT}$ values of the target gene to achieve the $\Delta \mathrm{CT}$ value. $2^{-\Delta \mathrm{CT}}$ was calculated for each sample and then each of the values was then divided by a control sample to achieve the relative miRNA levels $\left(2^{-\Delta \Delta C T}\right)$.

Target genes selected. Target genes were selected from the following websites: miRBase (http://microrna.sanger. ac.uk/sequences/), TargetScan (http://www.targetscan.org/), PicTar (http://pictar.mdc-berlin.de/) and miRanda (http://www. microrna.org/microrna/home.do).

\section{Results}

Establishment of a stably HBx-transfected LO2 cell line. RT-PCR was used to identify the integrated HBx gene in the cDNA of the engineered LO2 cells. $\beta$-actin was used as a loading control (Fig. 1A). The data showed that the HBx gene had been successfully introduced into the host genome of LO2 cells. Western blot analysis showed that expression of the HBx protein was detectable in LO2 cells (Fig. 1B). Our data suggest that a stably HBx-transfected LO2 cell line was successfully established.

RNA quality check. The mirvana miRNA isolation kit was used to extract and purify miRNA and the quality of RNA was analyzed using capillary electrophoresis individually. There were 3 visible electrophoresis strip peaks representing $5 \mathrm{~s}, 18 \mathrm{~s}$ and 28s, respectively (Fig. 2). Our data showed that the quality of RNA was high without degradation, and it was not polluted by genomic DNA (Table I). 
Table II. Differential miRNA expression in HBx-d382-transfected LO2 cells.

\begin{tabular}{lcccc}
\hline miRNA name & L02/PCDNA3.0 mean & L02/HBx-d382 mean & $\begin{array}{c}\text { Fluorescence difference } \\
\text { value (log2 value) }\end{array}$ & $\begin{array}{c}\text { Up- or downregulation } \\
\text { in L02/HBx-d382 cells }\end{array}$ \\
\hline hsa-miR-338-3p & 4.629886 & 1.577917 & -3.05196969 & Downregulated \\
hsa-miR-551b & 4.000756 & 1.198526 & -2.802229496 & Downregulated \\
hsa-miR-1 & 3.541191 & 1 & -2.541190817 & Downregulated \\
hsa-miR-455-3p & 5.435329 & 4.10132 & -1.334009537 & Downregulated \\
hsa-miR-200c & 3.309559 & 2.003005 & -1.306553869 & Downregulated \\
hsa-miR-501-5p & 2.094981 & 3.116949 & 1.021967456 & Upregulated \\
hsa-miR-1307 & 1.764127 & 2.865505 & 1.101377863 & Upregulated \\
hsa-miR-1180 & 1.697469 & 2.909304 & 1.211835771 & Upregulated \\
hsa-miR-497 & 1.653463 & 2.965216 & 1.31175287 & Upregulated \\
hsa-miR-1246 & 4.304869 & 5.661847 & 1.356977617 & Upregulated \\
hsa-miR-623 & 1.815217 & 3.272093 & 1.456875568 & Upregulated \\
\hline
\end{tabular}

A
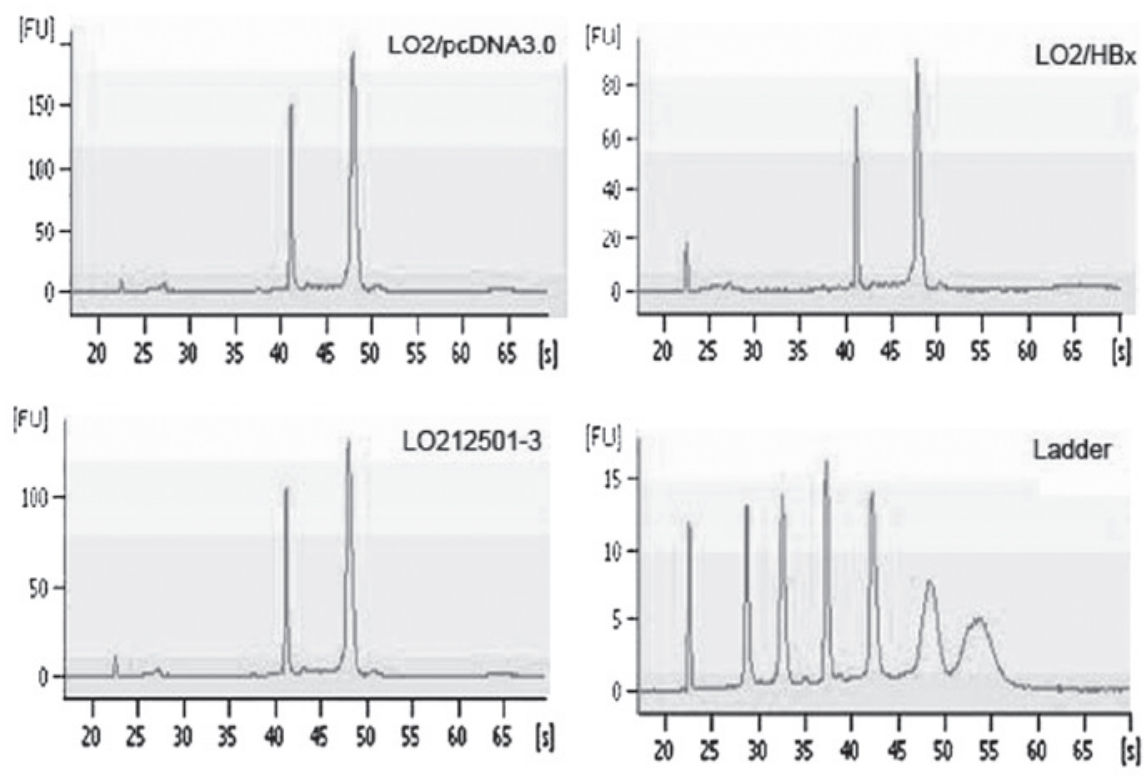

B

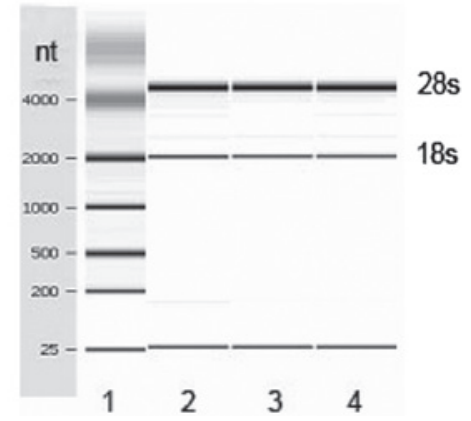

Figure 2. (A) The capillary electrophoresis of RNA in transfected cells. There were 3 visible electrophoresis strip peaks representing 5 (left), 18 (middle) and $28 \mathrm{~s}$ (right), respectively. The ladder was RNA marker. (B) Representative images of RNA electrophoresis. 1, LO2; 2, LO2/pcDNA3.0; 3, LO2/HBx-d382; 4, LO2/HBx.

miRNA microarray scanning. Fig. 3 shows images scanned with GenePix ${ }^{\mathrm{TM}}$ 4000B. The detection rate of all chips was $22.10 \pm 3.08 \%$, while the coefficient of variation $(\mathrm{CV})$ of one single sample was $8.93 \pm 2.11 \%$, which indicated that different measures of hybridization quality and consistency were high.
miRNA microarray analysis. Eleven miRNAs were differentially expressed in $\mathrm{LO} 2 / \mathrm{HBx}-\mathrm{d} 382$ compared to LO2/pcDNA3.0 cells. The normalized hybridization signal of miRNAs identified in the microarray analysis is listed in Table II. The 6 upregulated miRNAs were miR-501, 
Table III. Differential miRNA expression in HBx-transfected LO2 cells.

\begin{tabular}{|c|c|c|c|c|}
\hline miRNA name & L02/PCDNA3.0 mean & L02/HBx mean & $\begin{array}{c}\text { Fluorescence difference } \\
\text { value (log2 value) }\end{array}$ & $\begin{array}{l}\text { Up- or downregulation } \\
\text { in L02/HBx cells }\end{array}$ \\
\hline hsa-miR-338-3p & 4.629886 & 2.557788 & -2.0721 & Downregulated \\
\hline hsa-miR-24-1 & 3.602923 & 1.588591 & -2.01433 & Downregulated \\
\hline hsa-miR-200c & 3.309559 & 1.359513 & -1.950046 & Downregulated \\
\hline hsa-miR-29c & 8.110281 & 6.527961 & -1.58232 & Downregulated \\
\hline hsa-miR-744 & 3.99746 & 2.519724 & -1.47774 & Downregulated \\
\hline hsa-miR-455-3p & 5.435329 & 4.121858 & -1.31347 & Downregulated \\
\hline hsa-miR-324-5p & 5.94758 & 4.638507 & -1.30907 & Downregulated \\
\hline hsa-miR-551b & 4.000756 & 2.719708 & -1.28105 & Downregulated \\
\hline hsa-miR-340 & 3.018177 & 1.751115 & -1.26706 & Downregulated \\
\hline hsa-miR-590-5p & 4.962567 & 3.78986 & -1.17271 & Downregulated \\
\hline hsa-miR-660 & 4.119494 & 2.947035 & -1.17246 & Downregulated \\
\hline hsa-miR-193a-3p & 5.329816 & 4.214547 & -1.11527 & Downregulated \\
\hline hsa-miR-7 & 6.283198 & 7.473472 & 1.190274 & Upregulated \\
\hline hsa-miR-1274a & 6.674666 & 7.974562 & 1.299896 & Upregulated \\
\hline hsa-miR-137 & 1.788507 & 3.308352 & 1.519845 & Upregulated \\
\hline hsa-miR-663 & 1.124881 & 3.014342 & 1.889461 & Upregulated \\
\hline
\end{tabular}

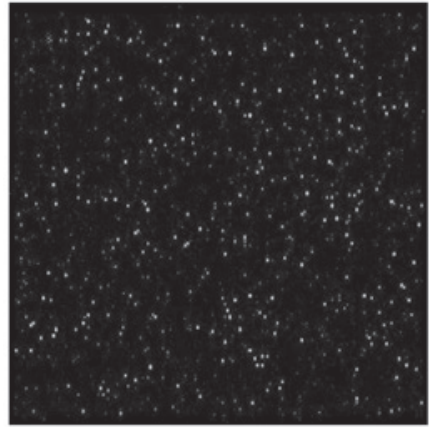

L02/pCDNA3.0

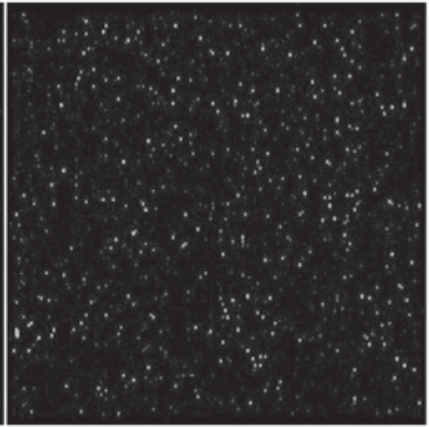

LO2/HBX

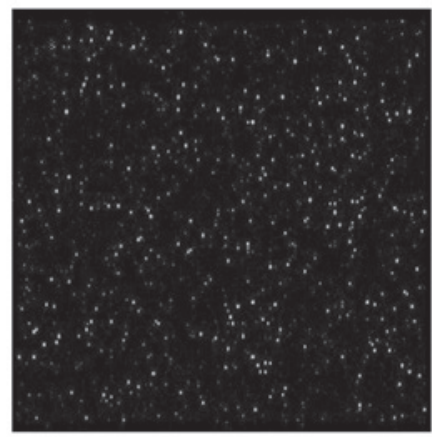

L02/HBX-d382

miR-1307, miR-1180, miR-497, miR-1246 and miR-623. The 5 downregulated miRNAs were miR-1, miR-338-3p, miR-551b, miR-455-3p and miR-200c.

Four upregulated and 12 downregulated miRNAs were observed in LO2/HBx compared to LO2/pcDNA3.0 cells. Normalized hybridization signals of miRNAs identified in the microarray analysis are listed in Table III. miR-7, miR-1274a, miR-137 and miR-663 were upregulated in LO2/HBx cells,

while miR-338-3p, miR-24-1, miR-200c, miR-29c, miR-744, miR-455-3p, miR-324, miR-551b, miR-340, miR-590, miR-660 and miR-193a-3p were downregulated.

Eight miRNAs were differentially expressed in LO2/HBx compared to LO2/HBx-d382 cells. Normalized hybridization signals of miRNAs identified in the microarray analysis are listed in Table IV. miR-551b, miR-663, miR-7 and miR-1 were upregulated and miR-1307, miR-501-5p, miR-29c and miR-24-1 were downregulated in LO2/HBx cells.

Real-time PCR analysis. The microarray data were confirmed by real-time PCR with the $2^{-\Delta \Delta C T}$ method. The whole miRNA expression of the HBx-expressed cell lines (LO2/HBx-d382 or $\mathrm{LO} 2 / \mathrm{HBx}$ ) were compared to each other as well as to LO2/pcDNA3.0 cells and normalized using U6 snRNA. The relative quantity of the control was normalized as 1 and, therefore, $2^{-\triangle \Delta C T}$ showed the relative miRNA levels. Our data revealed that the real-time PCR results were consistent with the chip results (Table V).

As shown in Table VI, the targets of miRNAs were predicted on several websites such as miRBase (http://microrna.sanger. ac.uk/sequences/), TargetScan (http://www.targetscan.org/), PicTar (http://pictar.mdc-berlin.de/) and miRanda (http://www. microrna.org/microrna/home.do).

\section{Discussion}

HCC is one of the most common malignancies and most of the cases are attributable to persistent HBV infection (14). Comparative studies of mammalian and avian hepadnaviruses, transgenic animal studies, and cell culture transformation studies imply that the $17.0-\mathrm{kDa} \mathrm{X}$ protein of $\mathrm{HBV}$ plays an important role in HCC development by influencing transcription, cell proliferation, apoptosis and signal transduction $(15,16)$. It has been reported that in tumor tissues, the majority of $\mathrm{HBx}$ natural mutants have lost their capacity for controlling cell 
Table IV. Eight miRNAs were differentially expressed in LO2/HBx compared to LO2/HBx-d382 cells.

\begin{tabular}{lcccc}
\hline miRNA name & L02/HBx-d382 mean & L02/HBx mean & $\begin{array}{c}\text { Fluorescence difference } \\
\text { value (log2 value) }\end{array}$ & $\begin{array}{c}\text { Up- or downregulation } \\
\text { in L02/HBx cells }\end{array}$ \\
\hline hsa-miR-551b & 1.198526 & 2.719708 & 1.521182 & Upregulated \\
hsa-miR-1307 & 2.865505 & 1.072718 & -1.79279 & Downregulated \\
hsa-miR-501-5p & 3.116949 & 1.199773 & -1.91718 & Downregulated \\
hsa-miR-29c & 7.846427 & 6.527961 & -1.31847 & Downregulated \\
hsa-miR-663 & 1.415526 & 3.014342 & 1.598816 & Upregulated \\
hsa-miR-7 & 6.44578 & 7.473472 & 1.027692 & Upregulated \\
hsa-miR-24-1 & 3.565019 & 1.588591 & -1.97643 & Downregulated \\
hsa-miR-1 & 1 & 2.906131 & 1.90613 & Upregulated \\
\hline
\end{tabular}

proliferation, viability and transformation and, therefore, may cause uncontrolled growth and contribute to the multistep development of HCC (17). A recent study demonstrated that a number of miRNAs have multiple functions including cell cycle control, cancer development, diagnosis and treatment (18-21). Given the importance of miRNAs in carcinogenesis, we hypothesized that miRNAs may also be involved in the mechanism of wild-type or mutant $\mathrm{HBx}$ in hepatocarcinogenesis.

In the present study, we established LO2 cell lines transfected with HBx-d382 and HBx. RT-PCR and western blot analysis showed that expression of the HBx gene was detectable in $\mathrm{LO} 2$ cells, a fact suggesting that stably HBx-transfected LO2 cell lines were successfully established. The miRNA expression profile was detected using the Agilent miRNA microarray. The results showed that 6 miRNAs exhibited higher expression and 5 miRNAs exhibited lower expression in LO2/HBx-d382 compared to LO2/pcDNA3.0 cells. Meanwhile, 4 upregulated and 12 downregulated miRNAs were observed in LO2/HBx compared to LO2/pcDNA3.0 cells. While of the above-mentioned miRNAs, miR-551b, miR-663, miR-7 and miR-1 were upregulated and miR-1307, miR-501-5p, miR-29c and miR-24-1 were downregulated in $\mathrm{LO} 2 / \mathrm{HBx}$ cells compared with $\mathrm{LO} 2 / \mathrm{HBx}-\mathrm{d} 382$ cells. Microarray data were consistent with the real-time qPCR analysis. Our data showed that the wild-type or mutant HBx had an impact on the miRNA expression of hepatic LO2 cells.

Of the above-mentioned miRNAs, the function of some has been revealed, while the function of others remains unknown. For example, miR-1 regulated aspects of both pre- and post-synaptic function at neuromuscular junctions. It regulated the muscle transcription factor MEF-2, which results in altered pre-synaptic acetylcholine (Ach) secretion (22). Other studies also found that miR-1 was involved in many types of cancer such as lung, colon and liver cancer, where it was expressed at a lower level $(23,24)$. Datta et al $(25)$ suggested that ectopic expression of miR-1 in HCC cells inhibited cell growth, reduced replication potential and clonogenic survival by regulating Foxp1 and MET and it was suggested to be one of the mechanisms by which DNA hypomethylating agents suppress hepatocarcinoma cell growth. From several predicted target websites, we found that there were many predicted targets of miR-1 and some of them were involved in cell proliferation or controlling the cell cycle, such as cyclin D1, cyclin D2, cdk-6 and cdk-9. A study showed that HBx induced DNA hypermethylation of the p16INK4a promoter to repress its expression, which subsequently led to activation of G1-CDKs, phosphorylation of $\mathrm{pRb}$, activation of E2F1, transcriptional activation of DNMT1 and finally induced development of HCC (26). Hence, we hypothesized that the significantly low expression of miR-1 in LO2/HBx-d382 cells may regulate cyclin D1 and induce cell growth.

Another widely-investigated miRNA is miR-200c. Yu et al (27) reported that miR-200c is an independent prognostic factor in pancreatic cancer and its upregulation inhibits pancreatic cancer invasion. They also found that the patients with high levels of miR-200c expression had significantly better survival rates than those with low levels of miR-200c expression. Several studies have shown that miR-200 family miRNAs (miR-200a, -200b, -200c-141 and -429) prevent epithelial-to-mesenchymal transition (EMT) by directly suppressing the expression of ZEB1 and ZEB2, causing degradation of the mRNA, and resulting in an upregulation of E-cadherin $(28,29)$. Previous studies have demonstrated that HBx regulated EMT in the SMMC-7721 hepatoma cell line in vivo (30). Moreover, our data indicated that a decrease of miR-200c expression was detected in both LO2/HBx-d382 and LO2/HBx cells, so we suggest that miR-200c is possibly linked to the mechanism of HBx in EMT. It has been recently reported that loss of miR200c expression is linked to aberrant DNA methylation and histone modifications, while HBx regulates DNA methylation (31). Therefore, further investigation is required to clarify whether miR-200c plays a key role in the upregulation of DNA methylation influenced by HBx.

miR-29c, a different isoform of miR-29, has been reported to target the expression of TCL1, a critical oncogene in aggressive chronic lymphocytic leukemia (CLL), a fact strongly suggesting that miR-29 may function as a tumor suppressor in CLL (32). A recent study showed that miR-29 directly targeted DNA methyltransferases Dnmt3A and -3B55 and that it activated P53 by targeting P85 $\alpha$ and CDC42 (33). Although there are insufficient data regarding its function, miR-338-3p has been detected by using a bead-based microarray analysis to be significantly downregulated in HCC tissue and this has been suggested to be related to the development of $\operatorname{HCC}(21,34)$. A number of miRNAs affect the growth of cancer cells in vitro and in vivo when overexpressed or inhibited. Therefore, cancer 
Table V. Relative miRNA expression levels.

\begin{tabular}{|c|c|c|c|}
\hline miRNA name & $\Delta \mathrm{CT}_{\text {sample }}$ & $\Delta \mathrm{CT}_{\text {control }}$ & $2^{-\Delta \Delta C T^{a}}$ \\
\hline \multicolumn{4}{|c|}{$\Delta \mathrm{CT}_{\text {sample }}=\left(\mathrm{CT}_{\text {target }}{ }^{-} \mathrm{CT}_{\mathrm{U} 6}\right)_{\mathrm{LO} / \mathrm{HBx}-\mathrm{d} 382 \text { cells }}$} \\
\hline \multicolumn{4}{|c|}{$\Delta \mathrm{CT}_{\text {control }}=\left(\mathrm{CT}_{\text {target }}-\mathrm{CT}_{\mathrm{U} 6}\right)_{\mathrm{LO} / \mathrm{pcDNA} 3.0 \text { cells }}$} \\
\hline hsa-miR-338-3p & 10.89 & 8.079 & 0.14 \\
\hline hsa-miR-551b & 12.83 & 10.18 & 0.16 \\
\hline hsa-miR-1 & 13.89 & 11.69 & 0.22 \\
\hline hsa-miR-455-3p & 13.22 & 11.94 & 0.41 \\
\hline hsa-miR-200c & 9.83 & 7.82 & 0.24 \\
\hline hsa-miR-501-5p & 10.99 & 12.14 & 2.2 \\
\hline hsa-miR-1307 & 8.50 & 9.64 & 2.2 \\
\hline hsa-miR-1180 & 8.64 & 9.84 & 2.3 \\
\hline hsa-miR-497 & 13.47 & 14.92 & 2.73 \\
\hline hsa-miR-1246 & 11.07 & 12.85 & 3.43 \\
\hline hsa-miR-623 & 9.1 & 11.38 & 4.85 \\
\hline \multicolumn{4}{|c|}{$\Delta \mathrm{CT}_{\text {sample }}=\left(\mathrm{CT}_{\text {target }}-\mathrm{CT}_{\mathrm{U} 6}\right)_{\mathrm{LO} / \mathrm{HBx} \text { cells }}$} \\
\hline \multicolumn{4}{|c|}{$\Delta \mathrm{CT}_{\text {control }}=\left(\mathrm{CT}_{\text {target }}-\mathrm{CT}_{\mathrm{U} 6}\right)_{\mathrm{LO} 2 / \mathrm{pcDNA} 3.0 \text { cells }}$} \\
\hline hsa-miR-338-3p & 9.87 & 7.60 & 0.21 \\
\hline hsa-miR-24-1* & 16.71 & 15.56 & 0.45 \\
\hline hsa-miR-200c & 10.10 & 8.78 & 0.4 \\
\hline hsa-miR-29c & 8.43 & 6.49 & 0.26 \\
\hline hsa-miR-744 & 12.72 & 10.91 & 0.28 \\
\hline hsa-miR-455-3p & 13.22 & 11.94 & 0.41 \\
\hline hsa-miR-324-5p & 13.83 & 11.70 & 0.23 \\
\hline hsa-miR-551b & 11.57 & 10.43 & 0.45 \\
\hline hsa-miR-340 & 14.98 & 13.90 & 0.47 \\
\hline hsa-miR-590-5p & 13.17 & 11.26 & 0.27 \\
\hline hsa-miR-660 & 8.56 & 7.45 & 0.46 \\
\hline hsa-miR-193a-3p & 15.63 & 13.87 & 0.30 \\
\hline hsa-miR-7 & 15.24 & 16.55 & 2.48 \\
\hline hsa-miR-1274a & 8.49 & 10.15 & 3.16 \\
\hline hsa-miR-137 & 7.13 & 8.86 & 3.32 \\
\hline hsa-miR-663 & 11.23 & 13.69 & 5.5 \\
\hline \multicolumn{4}{|c|}{$\Delta \mathrm{CT}_{\text {sample }}=\left(\mathrm{CT}_{\text {target }}-\mathrm{CT}_{\mathrm{U} 6}\right)_{\mathrm{LO} / \mathrm{HBx} \text { cells }}$} \\
\hline \multicolumn{4}{|c|}{$\Delta \mathrm{CT}_{\text {control }}=\left(\mathrm{CT}_{\text {target }}-\mathrm{CT}_{\mathrm{U} 6}\right)_{\mathrm{LO} / \mathrm{HBx}-\mathrm{d} 382 \text { cells }}$} \\
\hline hsa-miR-551b & 11.78 & 13.10 & 2.50 \\
\hline hsa-miR-1307 & 10.13 & 8.82 & 0.40 \\
\hline hsa-miR-501-5p & 11.69 & 10.41 & 0.41 \\
\hline hsa-miR-29c & 7.97 & 6.81 & 0.45 \\
\hline hsa-miR-663 & 11.06 & 12.95 & 3.71 \\
\hline hsa-miR-7 & 14.96 & 16.02 & 2.08 \\
\hline hsa-miR-24-1* & 17.00 & 15.28 & 0.30 \\
\hline hsa-miR-1 & 12.42 & 14.06 & 3.11 \\
\hline
\end{tabular}

${ }^{\mathrm{a}}$ When $2^{-\Delta \Delta \mathrm{CT}}>2$ or $<0.5$, the difference was considered to be significant.

cell growth may be controlled by manipulating miRNAs. For example, miR-7 and miR-137 are considered as cancer therapeutic tools (35). Silber et al (36) reported that the targeted delivery of miR-137 to glioblastoma multiforme tumor cells, which inhibited the proliferation of glioblastoma multiforme cells and induced differentiation of brain tumor stem cells, may be therapeutically effective for the treatment of this disease.
Our data showed that miRNA expression was different between the wild-type and mutant HBx-transfected LO2 cells. For example, miR-338-3p was detected to have a lower expression level in $\mathrm{LO} 2 / \mathrm{HBx}-\mathrm{d} 382$ compared to $\mathrm{LO} 2 / \mathrm{HBx}$ cells. On the contrary, miR-29c and miR-24-1 were detected to have a lower expression level in $\mathrm{LO} 2 / \mathrm{HBx}$ compared to LO2/HBx-d382 cells. miR-1 had a low expression level in 
Table VI. Predicted targets of miRNAs.

\begin{tabular}{|c|c|c|}
\hline miRNA name & Putative target associate with $\mathrm{HBx}$ & Description \\
\hline hsa-miR-338-3p & CCND (cyclin D1) & G1/S-specific cyclin D1 \\
\hline hsa-miR-551b & BCL2 & Apoptosis regulator $\mathrm{Bcl}-2$ \\
\hline \multirow[t]{4}{*}{ hsa-miR-1 } & CCND1 & \\
\hline & CCND2 & G1/S-specific cyclin D1 \\
\hline & cdk-6 & Cell division protein kinase 6 \\
\hline & cdk-9 & Cell division protein kinase 9 \\
\hline \multirow[t]{2}{*}{ hsa-miR-200c } & JUN & Transcription factor AP-1 (activator protein 1) \\
\hline & MYC & Myc proto-oncogene protein (c-Myc) \\
\hline \multirow[t]{2}{*}{ hsa-miR-501-5p } & HBXIP & Hepatitis B virus $\mathrm{X}$-interacting protein \\
\hline & RSF1 & $\begin{array}{l}\text { Remodeling and spacing factor } 1 \text { (Rsf-1) } \\
\text { (Hepatitis B virus X-associated protein) }\end{array}$ \\
\hline \multirow[t]{3}{*}{ hsa-miR-497 } & CCNE1 & G1/S-specific cyclin E1 \\
\hline & BCL2 & Apoptosis regulator $\mathrm{Bcl}-2$ \\
\hline & CCND2 & G1/S-specific cyclin D2 \\
\hline \multirow[t]{2}{*}{ hsa-miR-623 } & GCK & Glucokinase \\
\hline & CCRK & Cell cycle-related kinase \\
\hline hsa-miR-24-1 & BCL2L2 & Apoptosis regulator Bcl-W (Bcl-2-like 2 protein) \\
\hline \multirow[t]{2}{*}{ hsa-miR-29c } & PTEN & Phosphatase and tensin homolog deleted on chromosome 10 \\
\hline & PPP1R13B & Apoptosis-stimulating of $\mathrm{p} 53$ protein 1 \\
\hline \multirow[t]{3}{*}{ hsa-miR-324-5p } & GCK & \\
\hline & TMEM16J & Tumor proteinp 53 inducible protein 5 \\
\hline & CCND3 & G1/S-specific cyclin D3 \\
\hline hsa-miR-340 & CTNNB1 & Catenin $\beta-1$ ( $\beta$-catenin) \\
\hline \multirow[t]{3}{*}{ hsa-miR-193a-3p } & RSF1 & Remodeling and spacing factor 1 (Rsf-1) \\
\hline & PTEN & Phosphatase and tensin homolog deleted on chromosome 10 \\
\hline & APOA2 & Apolipoprotein A-II precursor (Apo-AII) \\
\hline \multirow[t]{3}{*}{ hsa-miR-7 } & APOA2 & \\
\hline & TP53INP2 & Tumor protein p53-inducible nuclear protein 2 \\
\hline & CKS2 & Cyclin-dependent kinases regulatory subunit 2 (CKS-2) \\
\hline \multirow[t]{2}{*}{ hsa-miR-137 } & CDK6 & Cell division protein kinase 6 \\
\hline & $\mathrm{CDC} 42$ & Cell division control protein 42 homolog precursor \\
\hline hsa-miR-663 & CDK3 & Cell division protein kinase 3 \\
\hline
\end{tabular}

LO2/HBx-d382 cells and no difference in LO2/HBx cells when compared to LO2/PCDNA3.0. As mentioned above, miR-1 had several predicted targets such as cyclin D1 and D2 which could promote cell proliferation, a fact which may explain why mutant HBx led to uncontrolled growth. Meanwhile, miR-551b, which demonstrated a lower expression level in LO2/HBx-d382 compared to LO2/HBx cells, may directly or indirectly regulate BCL2 (Table V), which is well-known as an anti-apoptotic protein (37). Therefore, the difference in miRNAs between the HBx-expressing cell lines may be one of the reasons for the different functions and biological characteristics of the wild-type and mutant HBx transfected cells.

Due to the fact that miRNA expression may differ from cells to tissues, and from hepatitis and cirrhosis to HCC, it also may be dissimilar in cell lines of different types of cancer as well as in cancer tissues resulting from different etiopathogenesis. Therefore, not all differential expression of miRNAs in our study could be the same as that in HCC tissue. However, due to the stable organization that is less variable than in tissue and unaffected by the immune system and the surrounding environment of stroma cells, we used cell lines to study the miRNA expression, which has certain advantages and is flexible. Our data showed that some of the differentially expressed miRNAs in LO2 cells transfected with wild-type or mutant HBx were similar to that in malignant tissues, suggesting that HBx may influence miRNA and, therefore, directly or indirectly induce hepatocarcinogenesis. Although the function of certain miRNAs found in this study has not been described, we could not exclude their potential involvement in oncogenesis. For example, although limited data were available regarding the function of miR-497, it was found to possess several predicted targets associated with $\mathrm{HBx}$ using public prediction algorithms, such as BCL2 and CCND2.

In conclusion, our study showed that the $\mathrm{X}$ gene is capable of influencing the miRNA expression profile in $\mathrm{LO} 2$ cells. Further investigation is required to clarify the roles of the identified miRNAs and, therefore, it may be helpful to inves- 
tigate the role of $\mathrm{HBx}$ in hepatocarcinogenesis as well as to clarify the underlying molecular mechanisms involved.

\section{Acknowledgements}

This study was supported by grants from the National Natural Science Foundation (no. 30872228), the National Key Program in the 11th Five Year Plan of China (no. 2008ZX10002-007), the Natural Science Foundation of Hunan (no. 10JJ5034) and Projects in the Science and Technology Pillar Program of Hunan (no. 2010SK3093).

\section{References}

1. Farazi PA and DePinho RA: Hepatocellular carcinoma pathogenesis: from genes to environment. Nat Rev Cancer 6: 674-687, 2006.

2. Parkin DM, Bray FI and Devesa SS: Cancer burden in the year 2000. The global picture. Eur J Cancer 37: S4-S66, 2001.

3. Wang WL, London WT, Lega L and Feitelson MA: HBxAg in the liver from carrier patients with chronic hepatitis and cirrhosis. Hepatology 14: 29-37, 1991.

4. Tang H, Oishi N, Kaneko S and Murakami S: Molecular functions and biological roles of hepatitis B virus x protein. Cancer Sci 97: 977-983, 2006

5. Sirma H, Giannini C, Poussin K, Paterlini P, Kremsdorf D and Bréchot C: Hepatitis B virus X mutants, present in hepatocellular carcinoma tissue abrogate both the antiproliferative and transactivation effects of HBx. Oncogene 18: 4848-4859, 1999.

6. Yu DY, Moon HB, Son JK, et al: Incidence of hepatocellular carcinoma in transgenic mice expressing the hepatitis B virus X-protein. J Hepatol 31: 123-132, 1999.

7. Liu XH, Lin J, Zhang SH, et al: $\mathrm{COOH}-$ terminal deletion of $\mathrm{HBx}$ gene is a frequent event in HBV-associated hepatocellular carcinoma. World J Gastroenterol 14: 1346-1352, 2008.

8. Ma NF, Lau SH, Hu L, et al: COOH-terminal truncated HBV X protein plays key role in hepatocarcinogenesis. Clin Cancer Res 14: 5061-5068, 2008.

9. Bartel DP: MicroRNAs: genomics, biogenesis, mechanism, and function. Cell 116: 281-297, 2004.

10. Budhu A, Jia HL, Forgues M, et al: Identification of metastasis-related micro-RNAs in hepatocellular carcinoma. Hepatology 47: 897-907, 2008.

11. Meng F, Henson R, Wehbe-Janek H, Ghoshal K, Jacob ST and Patel T: Micro-RNA-21 regulates expression of the PTEN tumor suppressor gene in human hepatocellular cancer. Gastroenterology 133: 647-658, 2007.

12. Huang J, Wang Y, Guo Y and Sun S: Downregulated microRNA-152 induces aberrant DNA methylation in hepatitis B virus-related hepatocellular carcinoma by targeting DNA methyltransferase 1. Hepatology 52: 60-70, 2010.

13. Zhu P, Tan D, Peng Z, Liu F and Song L: Polymorphism analyses of hepatitis B virus X gene in hepatocellular carcinoma patients from southern China. Acta Biochim Biophys Sin (Shanghai) 39: 265-272, 2007

14. Boyle P and Levin B: World Cancer Report 2008. International Agency for Research on Cancer, Lyons, France, pp 338-343, 2008.

15. Andrisani OM and Barnabas S: The role of the transcriptional involvement of $\mathrm{HBV} \mathrm{pX}$ in hepatocarcinogenesis (Review). Int $\mathrm{J}$ Oncol 15: 373-139, 1999.

16. Chen HY, Tang NH, Lin N, Chen ZX and Wang XZ: Hepatitis B virus $X$ protein induces apoptosis and cell cycle deregulation through interfering with DNA repair and checkpoint responses. Hepatol Res 38: 174-182, 2008

17. Chen S, Zhao W, Tan W, et al: Association of TBX21 T-1993C polymorphism with viral persistence but not disease progression in hepatitis B virus carrier. Hepatol Res 39: 716-723, 2009.

18. Carleton M, Cleary MA and Linsley PS: MicroRNAs and cell cycle regulation. Cell Cycle 6: 2127-2132, 2007.
19. Vebtura A and Jacka T: MicroRNAs and cancer: short RNAs go a long way. Cell 136: 586-591, 2009.

20. Masaki T: MicroRNA and hepatocellular carcinoma. Hepatol Res 39: 751-752, 2009

21. Huang XH, Wang Q, Chen JS, et al: Bead-based microarray analysis of microRNA expression in hepatocellular carcinoma: miR-338 is downregulated. Hepatol Res 39: 786-794, 2009.

22. Simon DJ, Madison JM, Conery AL, et al: The microRNA miR-1 regulates a MEF-2 dependent retrograde signal at neuromuscular junctions. Cell 133: 903-915, 2008.

23. Nasser MW, Datta J, Nuovo G, et al: Downregulation of micro-RNA-1 (miR-1) in lung cancer. Suppression of tumorigenic property of lung cancer cells and their sensitization to doxorubicin-induced apoptosis by miR-1. J Biol Chem 283: 33394-33405, 2008.

24. Sarver AL, French AJ, Borralho PM, et al: Human colon cancer profiles show differential MicroRNA expression depending on mismatch repair status and are characteristic of undifferentiated proliferative states. BMC Cancer 9: 401, 2009.

25. Datta J, Kutay H, Nasser MW, et al: Methylation mediated silencing of Micro-RNA-1 gene and its role in hepatocellular carcinogenesis. Cancer Res 68: 5049-5058, 2008.

26. Jung JK, Arora P, Pagano JS and Jang KL: Expression of DNA methyltransferase 1 is activated by hepatitis B virus $X$ protein via a regulatory circuit involving the p16INK4a-cyclin D1-CDK4/6-pRb-E2F1 pathway. Cancer Res 67: 5771-5778, 2007.

27. Yu J, Ohuchida K, Mizumoto K, et al: MicroRNA, hsa-miR-200c, is an independent prognostic factor in pancreatic cancer and its upregulation inhibits pancreatic cancer invasion but increases cell proliferation. Mol Cancer 9: 169, 2010.

28. Hurteau GJ, Carlson JA, Spivack SD and Brock GJ: Overexpression of the MicroRNA hsa-miR-200c leads to reduced expression of transcription factor 8 and increased expression of E-cadherin. Cancer Res 67: 7972-7976, 2007.

29. Park SM, Gaur AB, Lengyel E and Peter ME: The miR-200 family determines the epithelial phenotype of cancer cells by targeting the E-cadherin repressors ZEB1 and ZEB2. Genes Dev 22: 894-907, 2008

30. Yang SZ, Zhang LD, Zhang Y, et al: HBx protein induces EMT through c-Src activation in SMMC-7721 hepatoma cell line. Biochem Biophys Res Commun 382: 555-560, 2009.

31. Vrba L, Jensen TJ, Garbe JC, et al: Role for DNA methylation in the regulation of miR-200c and miR-141 expression in normal and cancer cells. PLoS One 5: e8697, 2010.

32. Pekarsky Y, Santanam U, Cimmino A, et al: Tcll expression in chronic lymphocytic leukemia is regulated by miR-29 and miR-181. Cancer Res 66: 11590-11593, 2006.

33. Fabbri M, Garzon R, Cimmino A, et al: MicroRNA-29 family reverts aberrant methylation in lung cancer by targeting DNA methyltransferases 3A and 3B. Proc Natl Acad Sci U S A 104: 15805-15810, 2007.

34. Park SY, Lee JH, Ha M, Nam JW and Kim VN: miR-29 miRNAs activate $\mathrm{p} 53$ by targeting p 85 alpha and CDC42. Nat Struct Mol Biol 16: 23-29, 2009

35. Kefas B, Godlewski L, Comeau L, et al: MicroRNA-7 inhibits the epidermal growth factor receptor and the Akt pathway and is downregulated in glioblastoma. Cancer Res 68: 3566-3572, 2008.

36. Silber J, Lim DA, Petritsch C, et al: miR-124 and miR-137 inhibit proliferation of glioblastoma multiforme cells and induce differentiation of brain tumor stem cells. BMC Med 6: 14, 2008.

37. Takada Y, Murakami A and Aggarwal BB: Zerumbone abolishes $N F-\kappa B$ and $I \kappa B \alpha$ kinase activation leading to suppression of antiapoptotic and metastatic gene expression, upregulation of apoptosis and downregulation of invasion. Oncogene 24: 6957-6969, 2005. 